\title{
VI. „Wir wollen der Sache einmal einen Auftrieb geben, wie es bisher... noch nie dagewesen ist "'. Zusammenfassung, Ergebnisse und Ausblick
}

Die Gründung der CSU im Herbst 1945 vollzog sich im „Spannungsfeld zwischen Tradition und Neuorientierung “2 . Die weitgehende territoriale Unversehrtheit und politisch-kulturelle Kontinuität Bayerns ermöglichte es den Frauen und Männern der ersten Stunde, an tief verwurzelte Traditionen anzuknüpfen, gemeinsame Sprachregelungen zu finden und die verstreuten Zirkel und Zentren der Unionsgründung vergleichsweise rasch zu einer landesweiten Organisation zusammenzufassen, sei es durch die Aktivierung persönlicher Kontakte aus der Zeit vor 1933 oder durch die Inanspruchnahme der staatlich-bürokratischen Infrastruktur, die bereits wenige Monate nach Kriegsende wieder zu funktionieren begann. Daß die Einheit Bayerns größtenteils erhalten blieb, eröffnete den Gründern der CSU jedoch nicht nur viele Chancen, sondern brachte auch unübersehbare Risiken mit sich. So drohte der in der NS-Zeit überlagerte, aber nicht überwundene Gegensatz zwischen den Protestanten in Ober- und Mittelfranken und den Katholiken in Altbayern, Schwaben und Unterfranken ebenso wieder aufzubrechen wie der Gegensatz zwischen den radikal föderalistischen, ja teilweise partikularistischen Kräften im Süden und Südosten Bayerns, die eine autonome bayerische Landespartei anstrebten, und der mehr gesamtdeutsch-national orientierten Bevölkerung in den Landesteilen, die erst zu Beginn des 19. Jahrhunderts an Bayern gefallen waren. Soweit die Menschen dort der CSU zuneigten, sahen sie in der neuen Partei weniger eine eigenständige Vertreterin bayerischer Interessen als einen Landesverband der künftigen „Reichsunion“.

Schon im Herbst 1945 prallten die divergierenden Standpunkte hart aufeinander; sollte die „bayerische Frage“ eher partikularistisch, föderalistisch oder zentralistisch gelöst werden? Hier erwies es sich als nahezu unmöglich, einen für alle Seiten akzeptablen Kompromiß zu finden. Schließlich sammelten sich in der CSU neben den Protagonisten der bayerischen Eigenstaatlichkeit auch gemäßigte Befürworter eines deutschen Bundesstaates oder Politiker, die mit dem Begriff Föderalismus nicht viel anfangen konnten. Die „bayerische Frage“ entwickelte sich schnell zum wichtigsten Katalysator der innerparteilichen Auseinandersetzungen, die die CSU bis 1949 wiederholt an den Rand des Zusammenbruchs brachten. Die Härte und Erbitterung, mit der die Meinungsverschiedenheiten über die Stellung Bayerns in einem übergeordneten deutschen Staatswesen ausgetragen wurden, wird nur dann verständlich, wenn man berücksichtigt, welchen enormen Stellenwert die „bayerische Frage“ und das bayerische Staatsbe-

\footnotetext{
' ACSP, CSU-LL, Protokoll der Sitzung des Landesausschusses der CSU am 18. 12. 1954 in München (Hans Ehard).

2 Mintzel, CSU, in: ders./Oberreuter (Hrsg.), Parteien in der BRD, S. 199.
} 
wußtsein insbesondere in den Regionen hatten, die jahrhundertelang den Kern des Kurfürstentums und später des Königreichs Bayern gebildet hatten.

Allen historischen und politischen Hypotheken zum Trotz gab es in den verschiedenen Gründungszirkeln freilich auch eine Reihe von Gemeinsamkeiten, die es erst möglich machten, daß die CSU - wenn auch unter schmerzhaften Geburtswehen - zu einer Partei zusammenwuchs. Angesichts der Führungs- und Flügelkämpfe, von denen die Ära Müller gekennzeichnet war, verschwanden diese Gemeinsamkeiten aber mehr und mehr aus dem Bewußtsein der Zeitgenossen und blieben auch in der Forschung häufig unberücksichtigt. $\mathrm{Da}$ war zunächst die in allen Gründungszirkeln verbreitete Angst vor einem politischen Linksrutsch. Diese Sozialismusfurcht und die Überzeugung, nur eine große Sammlungspartei aller nichtsozialistischen Kreise könne den Arbeiterparteien SPD und KPD erfolgreich Paroli bieten, ließen die Mitbegründer der CSU zusammenrücken, auch wenn sie vor 1933 unterschiedlichen politischen Lagern angehört hatten. Die totalitäre Erfahrung des Dritten Reiches, das Wissen um politische Verfolgung, die man oft genug am eigenen Leib zu spüren bekommen hatte, und der Widerstand gegen das NS-Regime waren ein weiteres wichtiges Bindeglied zwischen den Männern und Frauen, die die CSU 1945 ins Leben riefen. Von den 178 untersuchten Mitgliedern des (Erweiterten) Landesausschusses der CSU waren mindestens 95 mit dem nationalsozialistischen Herrschaftssystem in Konflikt gekommen; 61 von diesen CSU-Mitgliedern der ersten Stunde waren zwischen 1933 und 1945 zum Teil wiederholt verhaftet worden und für Wochen, Monate oder gar Jahre in den Gefängnissen und Konzentrationslagern des Dritten Reiches verschwunden. Daraus erwuchs in den Gründungstagen der CSU eine Art antinationalsozialistischer Impuls, der sich jedoch in der Folgezeit mehr und mehr abschwächte. Es gab zwar auch in den fünfziger Jahren noch Politiker wie Josef Müller oder Alois Hundhammer - hier ausnahmsweise einig -, die sich dagegen zur Wehr setzten, wenn belastete Personen in der CSU ein Amt übernehmen wollten. Je mehr sich das politische und gesellschaftliche Leben aber normalisierte, desto weniger spielte die Vergangenheit neuer Parteimitglieder eine Rolle. 1945 war die gemeinsame Erfahrung von Verfolgung und Widerstand für die Annäherung von katholischen und evangelischen Christen jedoch von kaum zu überschätzender Bedeutung und trug entscheidend dazu bei, daß das Mißtrauen und die Sprachlosigkeit zwischen den Konfessionen zumindest teilweise überwunden werden konnte. Bei vielen verfolgten Mitbegründern der CSU läßt sich zudem eine Abkehr von antidemokratischen, reaktionären oder autoritären Überzeugungen feststellen, die ihr politisches Handeln noch in der Weimarer Republik mitbestimmt hatten. Das heißt nicht, daß sie pluralistische, freiheitlich-demokratische Gesellschaftssysteme nun vorbehaltlos bejaht hätten, die Achtung von unveräußerlichen Grundund Menschenrechten, Rechtssicherheit und Rechtsstaatlichkeit gewannen jedoch einen bis dahin nicht gekannten Stellenwert. Die vergleichende Analyse früher CSUProgramme aus allen Teilen Bayerns ergab schließlich ein erstaunliches Maß an Übereinstimmung in Fragen der Kultur-, Wirtschafts- und Gesellschaftspolitik; diese programmatischen Gemeinsamkeiten wurden jedoch Zug um Zug von den ideologisierten Auseinandersetzungen um die „bayerische Frage“ überlagert.

Obwohl die historisch gewachsenen innerbayerischen Bruch- und Spannungslinien nach 1945 wieder aufbrachen, blieb die CSU nicht im altbayerisch-katholischen Raum gefangen. Anders als die BVP konnte die CSU die Grenzen der Traditionszonen 
überwinden und auch in den protestantischen Teilen Frankens viel stärker Fuß fassen, als es die bisherigen Forschungen suggerieren. In diesen Regionen hatte die CSU zwar nur wenige Mitglieder, sprach aber einen Teil der konservativen evangelischen Wähler an, die die BVP vor 1933 strikt abgelehnt hatten. Die großen Erfolge der CSU bei den ersten Wahlen waren überhaupt nur deshalb möglich. Die Parteiführung um Josef Müller versuchte alles, um die Union dauerhaft im fränkisch-protestantischen Raum zu verankern. So wurden etwa evangelische CSU-Politiker in den Führungsgremien der Partei stärker berücksichtigt, als es der nominellen Stärke des protestantischen Flügels entsprochen hätte: Im ersten Landesvorstand der CSU, der sich im Dezember 1946 konstituierte, betrug der Anteil der Protestanten mehr als 26 Prozent und deckte sich damit fast genau mit dem Anteil der Protestanten an der bayerischen Bevölkerung. Auch wenn die CSU schon 1948 in Ober- und Mittelfranken teilweise dramatische Einbrüche hinnehmen mußte, blieb sie in ihren Diaspora-Gebieten doch politisch präsent und war selbst in der Ära Ehard fränkischer und protestantischer, als es die BVP jemals gewesen war.

Die erbitterten inneren Konflikte, die auf einer Verflechtung von sachlichen, persönlichen und strukturellen Ursachen beruhten, waren bis 1949 zweifellos die größte Crux der CSU. Diese Auseinandersetzungen begleiteten die CSU praktisch vom ersten Tag an und waren für die Frühgeschichte der bayerischen Unionspartei geradezu konstitutiv. Es gab kaum eine Entscheidung, gleichgültig ob in Sach- oder Personalfragen, die nicht in irgendeiner Form von den Zwistigkeiten beeinflußt worden wäre. Die Flügelkämpfe zwischen Josef Müller und seinen Mitstreitern und ihren katholisch-konservativen Gegenspielern um Fritz Schäffer und Alois Hundhammer drohten die CSU nicht nur auf Landesebene zu paralysieren, sondern sie setzten sich bis auf die untersten Stufen der Parteiorganisation fort und machten eine effektive politische Arbeit auch dort oft unmöglich. Als besonders folgenschwer erwies sich der Antagonismus von Parteiführung und Landtagsfraktion. Die von den Gefolgsleuten Josef Müllers dominierten Spitzengremien der CSU versuchten zumeist vergeblich, Einfluß auf die Abgeordneten ihrer Partei in der Verfassunggebenden Landesversammlung und später im bayerischen Landtag auszuüben. Dagegen gelang es dem von der Fraktionsmehrheit unterstützten Alois Hundhammer zunehmend häufiger, Josef Müller das Leben schwer zu machen und die Fraktion zur zentralen Operationsbasis der innerparteilichen Opposition auszubauen. Landesausschuß und Landesversammlung konnten noch so elaborierte Programme oder Richtlinien für die Arbeit der Abgeordneten beschließen, die Fraktion wachte eifersüchtig über ihre Autonomie und handelte auch in entscheidenden Fragen immer wieder aus eigenem Ermessen. Diese Konstellation hatte nicht zu unterschätzende Folgen für die CSU als Partei. Das Gefühl der Ohnmacht ließ viele Delegierte und Aktivisten resignieren und erstickte in den Führungsgremien der CSU nach und nach den Willen, Demokratie und Gesellschaft aktiv mitzugestalten. Der Fraktion, das zeigte sich schon 1946 und 1947 bei der Bildung der ersten beiden Kabinette Ehard, wuchs mehr und mehr Macht zu, während die Partei, die Josef Müller als dynamische, eigenständige Kraft im politischen Prozeß verstanden hatte, zu einem bloßen „Hilfsorgan ${ }^{33}$ von Staatsregierung und Landtagsfraktion degenerierte.

\footnotetext{
${ }^{3}$ So Wulf Schönbohm, Die CDU wird moderne Volkspartei. Selbstverständnis, Mitglieder, Organisation und Apparat 1950-1980, Stuttgart 1985, S. 31, mit Blick auf die CDU in den fünfziger Jahren.
} 
Versucht man, die Führungs- und Flügelkämpfe zu periodisieren, bietet sich ein Drei-Phasen-Modell an. Die Monate bis zur ersten Landesversammlung der CSU im Mai 1946 standen im Zeichen hitziger Debatten über die Politik der BVP vor 1933, über ihre Haltung gegenüber dem aufkommenden Nationalsozialismus und über die Rolle, die das Führungspersonal der untergegangenen BVP in der Union spielen sollte. Die zweite Phase vom Mai 1946 bis zum Februar 1948 war dagegen ganz von den Auseinandersetzungen über die „bayerische Frage“ geprägt; die Rebellion des CSU-Bezirksverbands Oberbayern unter Fritz Schäffers Führung leitete die dritte Phase und zugleich das Ende der Ära Müller ein. 1948 kulminierten die inneren Konflikte in einer wahren Zerfallskrise der Partei. Die verlorenen Kommunalwahlen, der Aufstieg der Bayernpartei, der Zusammenbruch des Parteiapparats nach der Währungsreform und das finanzielle Desaster der neuen Parteizeitung Der Gerade Weg erschütterten die Partei bis an ihre Grundfesten und unterminierten die Stellung Josef Müllers mehr und mehr. Der katholisch-konservative Flügel um Alois Hundhammer hatte zwar nach wie vor keine Möglichkeit, einen eigenen Kandidaten für das Amt des Landesvorsitzenden durchzusetzen, mit Hans Ehard, dem bayerischen Ministerpräsidenten, gab es jedoch einen Politiker, der bei allen Gruppierungen der CSU gleichermaßen geachtet war und so beste Chancen hatte, Müller in einer Kampfabstimmung zu schlagen. Ehard drängte sich nicht nach diesem Amt, er empfand es als Bürde. Obwohl der Ministerpräsident von allen Seiten aufgefordert wurde, gegen Müller zu kandidieren, zögerte er lange, konnte sich des wachsenden Drucks aber schließlich nicht mehr erwehren. Am 28. Mai 1949 ließ sich der zaudernde Ehard von den Delegierten des Straubinger Parteitags auf den Schild heben. Die Ära Müller war damit unwiderruflich zu Ende.

Hans Ehard galt bereits seit längerer Zeit als Hoffnungsträger der bayerischen Unionspartei. Der Ministerpräsident schien als einziger in der Lage zu sein, den anhaltenden Querelen ein Ende zu setzen. Der gebürtige Franke gehörte nicht zu den radikal föderalistischen Hardlinern und war so für die nordbayerischen Anhänger Josef Müllers akzeptabel, in Altbayern honorierte man dagegen seinen beharrlichen Einsatz für bayerische Interessen. Auch in konfessioneller Hinsicht war Ehard nicht belastet. Er war weit davon entfernt, ein klerikaler Katholik zu sein, und seine Bekenntnisse zur interkonfessionellen Basis der Union waren durchaus glaubwürdig. Vor allem besaß Ehard jedoch eine Fähigkeit, die in der CSU anscheinend abhanden gekommen war: Probleme sachlich zu lösen und ohne persönliche Ressentiments konsensfähige Kompromißvorschläge auszuarbeiten. Seine Autorität als bayerischer Ministerpräsident und die Erfolge seiner föderalistischen Politik trugen ein übriges dazu bei, ihm die Unterstützung der Parteibasis zu sichern. Dennoch wurde Ehard nie ein Mann der Partei. Er war es nicht, als Josef Müller an der Spitze der CSU stand, und er wurde es auch nicht, nachdem er selbst den Landesvorsitz übernommen hatte. So kann es nicht überraschen, daß Ehard mit der Partei nur wenig anzufangen wußte; sie diente ihm überwiegend zur parlamentarischen Absicherung der Regierungsarbeit.

Ehards wichtigste Aufgabe war zweifellos, die innerparteilichen Auseinandersetzungen zu beenden. Tatsächlich stellte sich nach seiner Wahl eine erschöpfungsbedingte Beruhigung ein, die dann in eine oberflächliche Konsolidierung und einen instabilen Burgfrieden mündete. Zugespitzt könnte man auch sagen, daß die spannungsgeladene Atmosphäre der Führungs- und Flügelkämpfe mit der Zeit einer Art Friedhofsruhe 
wich. Dazu trug vor allem bei, daß viele Gefolgsleute Josef Müllers nach der Wahl Ehards in der CSU nicht mehr ihre Partei sahen, immer wieder überspielt wurden oder resigniert aus der Partei austraten. Diese Entwicklung schwächte die liberalen und die protestantischen Kräfte, die Programm und Politik der CSU in der Ära Müller mitgestaltet hatten, erheblich. Die Parteiführung beschwor die neue Einigkeit der Union geradezu gebetsmühlenhaft, obwohl die alten Frontlinien weiterhin bestanden, wenn die Streitigkeiten auch abgeflaut waren und zunehmend von neuen Konflikten überlagert wurden. So entzweiten sich die führenden CSU-Politiker immer wieder über der Frage nach dem richtigen Kurs gegenüber der Bayernpartei, die sich seit $1948 \mathrm{zu}$ einer gefährlichen landespolitischen Konkurrentin entwickelt hatte. Alois Hundhammer und Fritz Schäffer plädierten für eine möglichst weitgehende Zusammenarbeit mit der Bayernpartei, die sie im Kern für eine abtrünnige Schwesterpartei der CSU hielten und deren radikal föderalistischen Parolen sie eine Zeit lang näher standen als den Positionen der eigenen Partei. Der Realpolitiker Ehard stand der Bayernpartei und ihrem unberechenbaren Landesvorsitzenden Joseph Baumgartner aber höchst skeptisch gegenüber und lehnte Koalitionen auf Bundes- oder Landesebene ebenso ab wie alle Initiativen, die auf eine Verschmelzung der beiden Parteien unter Aufgabe des Unionsgedankens abzielten. Wollte Ehard die Einheit der CSU wahren, so konnte er kaum anders handeln. Eine Fusion von CSU und Bayernpartei, wie sie Hundhammer lange Zeit vorschwebte, hätte vermutlich die Spaltung der bayerischen Unionspartei bedeutet.

Seitdem sich 1949 mit der CSU-Landesgruppe in Bonn ein neuer Machtfaktor im organisatorischen Gefüge der Partei herausgebildet hatte, war auch das schwierige Verhältnis von Bundes- und Landespolitik eine ständige Quelle kleinerer und größerer Reibereien. Unter der entschlossenen Führung von Fritz Schäffer und Franz Josef Strauß hatte sich die Landesgruppe rasch etabliert und begann, die innerparteiliche Willensbildung maßgeblich zu beeinflussen. Eine solche Entwicklung war freilich nicht im Sinne des Parteivorsitzenden und Ministerpräsidenten. In Ehards Kalkül spielte die Landesgruppe lediglich als föderalistisches Sprachrohr und Vollzugsorgan von Staatsregierung und Parteiführung eine Rolle. Die Landesgruppe wachte jedoch voller Argwohn über ihre Autonomie, verbat sich wiederholt Einmischungsversuche und gestattete sich das Recht auf eine eigene Meinung. Die daraus resultierenden Konflikte hatten ihren Grund nicht zuletzt in der Tatsache, daß die Mehrheit der Bonner CSU-Abgeordneten ein anderes Föderalismusverständnis entwickelte als ihre Münchner Parteifreunde. Zwar trat auch die Landesgruppe für die Stärkung und Durchsetzung des föderativen Gedankens ein, die Bundestagsabgeordneten der CSU waren aber dennoch bereit, aus koalitionspolitischen Zwängen, übergeordneten parteipolitischen Interessen oder nationaler Verantwortung heraus Entscheidungen mitzutragen, die aus der Perspektive bayerischer Föderalisten unpopulär und gefährlich waren. Dies zeigte sich beispielsweise, als die Landesgruppe die Erhöhung des Bundesanteils an der Einkommen- und Körperschaftsteuer gegen erhebliche Widerstände aus der eigenen Partei unterstützte und damit den Bund auf Kosten der Länder stärkte. Während viele Landespolitiker der CSU in ihrer bayerischen Provinzialität gefangen und dem Erbe der BVP verhaftet blieben und sich auf die klassischen Themen Schul-, Kultur- und Agrarpolitik konzentrierten, besetzten junge Bundestagsabgeordnete wie Franz Josef Strauß, Werner Dollinger, Richard Jaeger, Gerhard Wacher oder Richard 
Stücklen neue Politikfelder und machten sich von belastenden Traditionen frei. So warnte die Landesgruppe wiederholt davor, allzu starr auf schul- und kulturpolitischen Maximalforderungen zu beharren, wie dies Alois Hundhammer und Georg Meixner 1954 im Landtagswahlkampf taten. Noch waren die Bundespolitiker nicht in der Lage, entscheidend in die Landespolitik einzugreifen, aber die Landesgruppe wurde mehr und mehr zur Speerspitze der reformbereiten Kräfte in der CSU, die nur auf ihre Stunde warteten.

Die Führungs- und Flügelkämpfe hatten die CSU in der Ära Müller zwar immer wieder vor schwere Zerreißproben gestellt, aber zu dieser Zeit war die CSU wenigstens eine lebendige Partei gewesen, auch wenn Diskussionen über Sach- und Personalfragen immer wieder in erbitterte Auseinandersetzungen ausgeartet waren. Bei allen - oft kleinlichen - Streitereien hatte man in den Führungsgremien der CSU doch immer um den richtigen Weg für den demokratischen Aufbau von Staat und Gesellschaft gerungen. In der Ära Ehard wurde dagegen Parteidisziplin großgeschrieben, und die Parteiführung war darum bemüht, kontroverse Fragen im kleinen Kreis zu entscheiden und so wenig wie möglich nach außen dringen zu lassen. Daß darunter auch die innerparteiliche Demokratie litt, liegt auf der Hand. Die Delegierten des Landesausschusses und der Landesversammlung, ja selbst viele Mitglieder des Parteivorstands waren an der innerparteilichen Willensbildung nur am Rande beteiligt. Ein unzufriedener Delegierter protestierte 1952 offen dagegen, daß der Landesausschuß nicht mehr wie in der Ära Müller die Politik mitbestimme, sondern nur noch eine schöne „Fassade“ sei und nichts zu sagen habe ${ }^{4}$. Wichtige Entscheidungen wurden zumeist in Gremien vorbereitet, die von der Satzung nicht vorgesehen waren, und erst dann den zuständigen Parteiinstanzen vorgelegt, wenn die Delegierten von Landesausschuß und Landesversammlung überhaupt befragt und nicht vor vollendete Tatsachen gestellt wurden. Die Flügelkämpfe der frühen Jahre scheinen die Diskussions- und Streitkultur in der CSU aber weit über die Ära Ehard hinaus geprägt zu haben und auch für viele spätere Spitzenpolitiker, die diese Auseinandersetzungen meist als junge Menschen miterlebt hatten, ein traumatisches Erlebnis gewesen zu sein. Das bis heute spürbare Bemühen der CSU, stets den Eindruck zu erwecken, als sei die Partei ein monolithischer Block, dürfte auch auf die Erfahrung der aller Kontrolle entglittenen innerparteilichen Konflikte der ersten Nachkriegsjahre zurückgehen.

Müßte man die CSU in der Ära Ehard mit zwei Worten beschreiben, so wären zweifellos Stagnation und Sklerose die passenden Begriffe. Nach der Wahl Hans Ehards war es zwar gelungen, den akuten Zerfallsprozeß zu stoppen, aber es folgte kaum mehr als eine Konsolidierung der Partei auf niedrigem Niveau. Der erwartete Aufbruch blieb ebenso aus wie die dringende Reorganisation der Partei an Haupt und Gliedern. Man startete zwar wiederholt Initiativen, um die Parteiorganisation zu aktivieren und aus den Resten des Parteiapparats, der nach der Währungsreform auf eine Handvoll schlecht ausgestatteter Geschäftsstellen geschrumpft war, etwas Neues aufzubauen. Alle Bemühungen blieben aber Stückwerk oder verliefen völlig im Sande. Innovative Konzepte fehlten ebenso wie zupackende Aktivisten, die sich dieser Aufgabe angenommen hätten. Am schwersten wog jedoch zweifellos der chronische Geldman-

4 ACSP, CSU-LL, Protokoll der Landesversammlung am 5./6. 7. 1952 in Regensburg (Delegierter Huber, München). 
gel, der die CSU zu einer Zeit, als die staatliche Finanzierung politischer Parteien noch in den Anfängen steckte, fast zur vollständigen Handlungsunfähigkeit verurteilte. Von außen betrachtet erinnerte die CSU zwischen 1949 und 1955 an die Bayerische Volkspartei. Wie die BVP hatte sie sich zu einer mitgliederschwachen Honoratiorenpartei entwickelt, die in einigen Regionen Bayerns nur zu den Wahlterminen präsent, ansonsten aber organisatorisch kaum verankert war. Bekannte BVP-Politiker hatten nach dem Sturz Josef Müllers zudem einflußreiche Positionen übernommen, von wo aus sie die Politik der CSU entscheidend prägten.

Die organisatorische und programmatische Stagnation der CSU, ihre Immobilität und Überalterung riefen vor allem bei den Vertretern der jüngeren Generation große Unzufriedenheit hervor, die erkannt hatten, daß diese Entwicklung in eine Sackgasse führen mußte. Doch die Vorstöße aus den Reihen der Landesgruppe und der Jungen Union verpufften in einer Atmosphäre der Selbstzufriedenheit und Selbstüberschätzung. Den reformbereiten Kräften in der CSU, die sich hinter der konservativen Fassade formiert hatten, waren noch die Hände gebunden. Es bedurfte erst einer fundamentalen Erschütterung der Partei, um ihnen den Weg zu ebnen.

Dieser „Wendepunkt in der Geschichte der CSU“5 war mit der Bildung der Viererkoalition unter Führung der SPD erreicht. Dabei hatte nach den Landtagswahlen vom 28. November 1954 nichts darauf hingedeutet, daß die bayerische Unionspartei den Weg in die Opposition würde antreten müssen. Im Gegenteil: Die CSU hatte 38,0 Prozent der Stimmen erhalten und war damit wieder zur mit Abstand stärksten Partei geworden, während SPD und FDP auf dem Niveau von 1950 verharrten und der BHE ebenso Verluste zu verzeichnen hatte wie die Bayernpartei ${ }^{6}$. Doch die führenden Politiker der CSU waren sich ihrer Sache zu sicher. Gerade Alois Hundhammer und Georg Meixner glaubten, aus einer Position der Stärke heraus den potentiellen Koalitionspartnern die Bedingungen für eine Regierungsbeteiligung diktieren zu können ${ }^{7}$. Überdies zog sich die Willensbildung in der CSU quälend lange hin, und Ehards Stellung als Parteivorsitzender war nicht mehr so unangefochten wie noch vier Jahre zuvor, als er gegen den entschiedenen Widerstand Hundhammers eine Koalition mit der SPD durchgesetzt hatte. In den Wochen vor den Landtagswahlen war sogar über einen möglichen Nachfolger spekuliert worden ${ }^{8}$ ! Während die CSU mit sich selbst beschäftigt war, nutzte Waldemar von Knoeringen, der Vorsitzende der bayerischen SPD, die Gunst der Stunde. Taktisch geschickt brachte er durch weitgehende Zugeständnisse in Sach- und Personalfragen eine für unmöglich gehaltene Koalition aus SPD, Bayernpartei, BHE und FDP zustande, wobei ihm vor allem der kulturpolitische Fundamentalismus von Hundhammer und Meixner entgegenkam. So wurde am 14. Dezember 1954 nicht Hans Ehard oder einer seiner Parteifreunde, sondern der Sozialdemokrat Wilhelm Hoegner mit 112 von 197 Stimmen zum Ministerpräsidenten gewählt.

5 Mintzel, Geschichte der CSU, S. 67.

61950 hatte die CSU nur 27,4 Prozent der Stimmen gewinnen können. Die SPD konnte ihren Wähleranteil 1954 geringfügig von 28,0 auf 28,1 Prozent verbessern, die FDP von 7,1 auf 7,2 Prozent. Der BHE kam auf 10,2 Prozent der Stimmen (1950: 12,5 Prozent) und die Bayernpartei nur noch auf 13,2 Prozent (1950: 17,9 Prozent). Zahlen bei Ritter/Niehuss, Wahlen in Deutschland, S. $174 \mathrm{f}$.

7 Zur Bildung des zweiten Kabinetts Hoegner vgl. Bretschneider, Viererkoalition.

${ }^{8}$ Christ und Welt vom 15.7. 1954: „Ein Ministerpräsident gesucht. Wer wird Bayerns kommender Mann?/ Wirtschaftsminister Seidel tritt in den Vordergrund“. 
Für die Enttäuschung und die Wut, die sich in der CSU ausbreiteten wie ein Lauffeuer, lassen sich kaum Worte finden. Die Funktionsträger und die Aktivisten an der Basis, die hart für den Wahlerfolg gearbeitet hatten, sahen sich durch das - in ihren Augen - völlige Versagen der Parteiführung um die Früchte ihres Einsatzes gebracht und übten massive Kritik an Persönlichkeiten wie Ehard, Hundhammer oder Meixner, die lange Zeit als unantastbar gegolten hatten. Scharfe Attacken gegen die "widernatürliche Koalition" von Ministerpräsident Hoegner und die "Auslieferung Bayerns an die Marxisten und Zentralisten "9 konnten nicht darüber hinwegtäuschen, daß es in der CSU brodelte. Landesvorstand und Landesausschuß kamen zu Krisensitzungen zusammen, und viele Delegierte plädierten für einen radikalen Schnitt und einen Neuanfang. Hans Ehard selbst war es, der am 18. Dezember 1954 vor dem Landesausschuß die Parole ausgab: „Wir wollen der Sache einmal einen Auftrieb geben, wie es bisher hoffentlich noch nie dagewesen ist." Und Franz Josef Strauß ergänzte:

"Wir reden nicht aus Verärgerung oder Verbitterung; denn die CSU war in Gefahr, in ständiger Gewöhnung an die für selbstverständlich gehaltene Tatsache, daß sie in der Regierung sitzt, etwas an politischer Kampfkraft einzubüßen. Sie war in Gefahr, möchte ich sagen, daß das Gleichgewicht der Kräfte bei uns gestört wurde zugunsten der etwas ruhigeren und etwas bequemeren Elemente in unseren Reihen. Das Schicksal [...] hat dafür gesorgt, daß wir aufgerüttelt worden sind. Der Fehdehandschuh ist hingeworfen worden, wir greifen ihn auf, um Bayerns und Deutschlands willen!"10

Daß Franz Josef Strauß zu denen gehörte, die den neuen Kurs der CSU bestimmen sollten, war ebenso klar wie die Tatsache, daß Ehard daran keinen entscheidenden Anteil mehr haben würde. Der abgelöste Regierungschef ließ sich am 13. Dezember 1954 zum Präsidenten des bayerischen Landtags wählen und stellte sein Amt als Landesvorsitzender zur Verfügung. Ehard übernahm das Amt des Landtagspräsidenten von Alois Hundhammer, der nicht nur für viele Abgeordnete aus den Reihen der Viererkoalition ein rotes Tuch war, sondern auch für einen Teil seiner eigenen Parteifreunde, die ihn für den Sturz in die Opposition mitverantwortlich machten ${ }^{11}$. Auch Georg Meixner blieb nicht ungeschoren. Die Landtagsfraktion wählte den Prälaten zwar erneut zu ihrem Vorsitzenden, schränkte aber seine Macht erheblich ein, indem sie gleichzeitig Hanns Seidel zum "Sprecher der Opposition“ bestimmte. Hanns Seidel entschied auch das Rennen um den Parteivorsitz für sich. Am 22. Januar 1955 setzte sich der ehemalige Wirtschaftsminister in einer außerordentlichen Landesversammlung mit 380 zu 239 Stimmen gegen Franz Josef Strauß durch. Auch Josef Müller und Fritz Schäffer hatten ihren Hut noch einmal in den Ring geworfen, ihre Kandidatur aber schließlich zurückgezogen, als sie erkannten, daß sie chancenlos sein würden. Ihre Zeit war vorbei ${ }^{12}$.

Mit Hanns Seidel trat ein Vertreter der liberal-konservativen, interkonfessionellen Linie an die Spitze der CSU. Der neue Parteichef hatte schon vor 1949 Josef Müller in seinem Kampf gegen die BVP-treuen Kräfte in der CSU unterstützt und wurde

9 ACSP, CSU-LTF III/1, 8-1/1, Flugblatt der CSU-Landesleitung „Bayerische Wähler habt Ihr das gewollt?".

10 ACSP, CSU-LL, Protokoll der Sitzung des Landesausschusses der CSU am 18.12. 1954 in München.

1 Der Staatssekretär im Bundesinnenministerium, Hans Ritter von Lex, schrieb am 14. 12. 1954 an Hilde Albert (BAK, NL Lex 3): „Die Regierungsbildung in Bayern haben Ehard und Hundhammer sich selbst zuzuschreiben, weil Hundhammer, berauscht vom Wahlerfolg seiner Partei, verkündet hatte, dass man jetzt sofort mit allem Nachdruck an die konfessionelle Lehrerbildung herangehen müsse."

12 Vgl. Groß, Hanns Seidel, S. 121-129. 
nun gleichsam der Vollstrecker von Müllers politischem Testament. In diesem Sinne wählte Seidel auch seine neue Führungsmannschaft aus. Franz Josef Strauß und Rudolf Eberhard, pragmatische, kompetente und energische Repräsentanten der jüngeren Generation, behielten ihre Ämter als stellvertretende Landesvorsitzende. Fritz Zimmermann, der JU-Politiker und ehemalige persönliche Referent Josef Müllers, übernahm die Leitung der Landesgeschäftsstelle. „Hanns Seidel als Vorsitzender, Franz Josef Strauß und Rudolf Eberhard als Stellvertreter und nicht Hundhammer, das war ein Programm “, erinnerte sich Zimmermann Jahre später ${ }^{13}$. Zweifellos war es die durch den Machtwechsel ausgelöste Krise, die die Neutralisierung des katholisch-konservativen Flügels der CSU begünstigte und die bislang blockierten Reformpotentiale freizusetzen half.

Ende 1954 begann ein beschleunigter innerparteilicher Elitenwechsel. Im Zuge dieses Prozesses gelangten zunehmend junge Politiker (während des Ersten Weltkriegs und später geboren) in Führungspositionen, deren politische Sozialisation sich erheblich von der jener Persönlichkeiten unterschied, die bereits in der Zwischenkriegszeit öffentliche Ämter und Funktionen bekleidet hatten. Karl Möckl hat überspitzt von Männern der „Frontgeneration“ gesprochen, die sich Zug um Zug zu „Technokraten der Macht“ entwickelt hätten ${ }^{14}$. Insbesondere der tatkräftige, bisweilen hemdsärmelige Fritz Zimmermann erwies sich als Glücksgriff. Gegen den Widerstand Alois Hundhammers, dem Zimmermanns Lebenswandel ebenso suspekt war wie seine politische Vergangenheit, und gegen die Bedenken Georg Meixners wurde der 29 jährige Jurist im Januar 1955 mit der Leitung der Parteizentrale beauftragt ${ }^{15}$. Der neue Hauptgeschäftsführer, der den Titel Generalsekretär erst ein Jahr später führen durfte, übernahm einen Parteiapparat, dessen Zustand man nur als desolat bezeichnen konnte. Zimmermann übertrieb nicht, als er in der Rückschau erklärte: „Die Partei war eine Wüste. " ${ }^{16}$ Die neue Parteiführung wußte nicht einmal, wieviele Mitglieder die CSU hatte. Es gab keine Mitgliederkartei, die Landesgeschäftsstelle war hoffnungslos unterbesetzt, die Kassen waren leer und einflußreiche Bezirks- und Kreisvorsitzende wachten eifersüchtig über die Autonomie ihrer ausgezehrten Duodezfürstentümer. Von koordinierter Öffentlichkeitsarbeit konnte keine Rede sein, und mit dem Bayern-Kurier, das hatte auch Hans Ehard erkannt, ließ sich kein „Hund vom Ofen“ locken ${ }^{17}$.

Als der von der Spielbankenaffäre schwer angeschlagene Zimmermann 1963 sein Amt an den neuen Generalsekretär Anton Jaumann übergab, hatte er Beachtliches geleistet. Die Zahl der Mitglieder, die zwischen 1948 und 1955 auf 35000 gesunken war, hatte sich auf mehr als 56000 erhöht $^{18}$. Die Landesgeschäftsstelle war nach einer grundlegenden Organisationsreform auf dem besten Weg, eine wirklich leistungsfähige Schaltzentrale zu werden, und auch die Situation in den Bezirksgeschäftsstellen hatte sich entscheidend verbessert. Zimmermanns vielleicht größte Leistung war es jedoch, daß er in den bayerischen Bundeswahlkreisen Geschäftsstellen aufbaute, deren Personal

${ }^{13}$ Erinnerungsinterview mit Friedrich Zimmermann, in: Geschichte einer Volkspartei, S. 623.

${ }^{14}$ Möckl, Struktur, S. 737; zur Bedeutung des Kriegserlebnisses und der „Kriegsgeneration“ vgl. Erinnerungsinterview mit Friedrich Zimmermann, in: Geschichte einer Volkspartei, S. $621 \mathrm{f}$.

15 ACSP, CSU-LL, Protokoll der Sitzung des geschäftsführenden Landesvorstands am 8. 1. 1955.

${ }_{16}$ Erinnerungsinterview mit Friedrich Zimmermann, in: Geschichte einer Volkspartei, S. 623.

17 ACSP, CSU-LL, Protokoll der Sitzung des Landesausschusses der CSU am 18. 12. 1954 in München.

18 Zahlen nach Mintzel, CSU, in: ders./Oberreuter (Hrsg.), Parteien in der BRD, S. 217. 
von der Landesleitung angestellt und besoldet wurde ${ }^{19}$. Damit verfügte die CSU erstmals seit der Währungsreform wieder über ein einigermaßen tragfähiges organisatorisches Gerüst. Beim Amtsantritt Anton Jaumanns befand sich die CSU in einer organisationspolitischen Übergangsphase. Auf Landes-, Bezirksverbands- und auf Bundeswahlkreisebene war das Honoratiorensystem zugunsten von hauptamtlich besetzten, vergleichsweise leistungsfähigen und gut ausgestatteten Geschäftsstellen zurückgedrängt worden. Die Kreis- und Ortsverbände dagegen hatte dieser Veränderungsprozeß noch kaum erfaßt - hier dominierten nach wie vor die überkommenen Strukturen ${ }^{20}$.

Die neue Führungsmannschaft um Hanns Seidel konnte bald die ersten Früchte ihrer Arbeit ernten. Im September 1957 erreichte die CSU bei den Bundestagswahlen mit 57,2 Prozent der Stimmen ein geradezu sensationelles Ergebnis. Dieses Resultat blieb nicht ohne Folgen. Schon wenige Wochen später zerbrach die Viererkoalition, und Hanns Seidel wurde am 16. Oktober von den Abgeordneten der CSU, der FDP und des BHE zum bayerischen Ministerpräsidenten gewählt ${ }^{21}$.

Bevor die CSU aber den Durchbruch zur dominierenden politischen Kraft Bayerns endgültig schaffen konnte, sah sich die Partei einer vierfachen Herausforderung gegenüber. Damit sind zum Teil langwierige Integrationsprozesse angesprochen, die auf grundlegende Entwicklungen der westdeutschen Gesellschaft und des westdeutschen Parteiensystems verweisen. So war die CSU, erstens, gezwungen, der Bayernpartei zu begegnen, zweitens, das große Potential der Flüchtlinge und Vertriebenen zu integrieren und, drittens, die Interessen des protestantischen Bevölkerungsteils Bayerns angemessen zu berücksichtigen, um dem Anspruch der Interkonfessionalität gerecht zu werden, den sie von Beginn an erhoben hatte. Konnte die CSU bis 1966 die bayerische Konkurrenzpartei aus dem Felde schlagen und auch den überwiegenden Teil der Flüchtlinge und Heimatvertriebenen auf ihre Seite ziehen ${ }^{22}$, so blieb der konfessionelle Konsens noch längere Zeit labil, wenn auch Konflikte dieser Art viel von der Brisanz früherer Tage verloren hatten. Seit 1970 war es der CSU auch zunehmend möglich, der vierten Herausforderung zu begegnen und immer mehr Wählerstimmen in den traditionellen fränkischen Hochburgen von SPD und FDP zu gewinnen. Dank dieses Durchbruchs erzielte die CSU 1970 mit 56,4 Prozent der Stimmen erstmals seit 1946 bei Landtagswahlen wieder die absolute Mehrheit, die sie vier Jahre später auf 62,1 Prozent steigern konnte ${ }^{23}$. Der CSU war es damit gelungen, „die konservative Bastion Bayern [...] zu einer geschlossenen Gesamt-Hochburg “ auszubauen ${ }^{24}$.

$\mathrm{Zu}$ den wesentlichen Aufgaben der CSU als Regierungspartei in München und Bonn gehörte zweifelsohne die politische Steuerung der „späten Vollindustrialisierung“25

19 Vgl. Mintzel, Anatomie, S. 301-313.

${ }^{20}$ Vgl. ebenda, S. $282 \mathrm{f}$.

21 Vgl. Groß, Hanns Seidel, S. 173-186.

${ }^{22}$ Die Bayernpartei scheiterte bei den Landtagswahlen von 1966, die parlamentarische Repräsentanz der Flüchtlingsparteien endete bereits mit den Landtagswahlen von 1962; vgl. Ritter/Niehuss, Wahlen in Deutschland, S. 174 f. Zur Absorption der Flüchtlinge und Vertriebenen durch die CSU vgl. Mintzel, CSU, in: Stöss (Hrsg.), Parteien-Handbuch, Bd. 2, S. 695. Nach der Volkszählung von 1961 waren rund 20 Prozent der Bevölkerung Bayerns Vertriebene (17,3 Prozent) oder Flüchtlinge aus der SBZ/ DDR (3,1 Prozent); Zahlen nach Helmut Hoffmann, Bayern. Handbuch zur staatspolitischen Landeskunde der Gegenwart, München 7., völlig überarbeitete Auflage 1981, S. 26.

${ }^{23}$ Vgl. Ritter/Niehuss, Wahlen in Deutschland, S. 174.

${ }^{24}$ Mintzel, CSU, in: ders./Oberreuter (Hrsg.), Parteien in der BRD, S. 227.

${ }^{25}$ Mintzel, Geschichte der CSU, S. 35. 
Bayerns und des damit verbundenen sozialen und ökonomischen Wandels. Der Freistaat gehörte zwar lange Zeit zu den strukturschwächsten westdeutschen Ländern, doch dafür wurde Bayern um so nachhaltiger von sozioökonomischen Prozessen erfaßt, die Hans-Peter Schwarz als "Vorgang des großen Abräumens" bezeichnet hat, der die Gesellschaft der Bundesrepublik „gewissermaßen bis auf die Gene durchindustrialisiert, technisiert und rationalisiert" habe ${ }^{26}$.

Wie tiefgreifend der soziale Wandel im Freistaat war, zeigt ein Blick auf die Erwerbsstruktur Bayerns: 1933 waren noch 41,2 Prozent der Beschäftigten in der Landwirtschaft tätig, 1950 noch immer 30,6 Prozent; bis 1961 reduzierte sich dieser Anteil auf 21,6 Prozent und neun Jahre später waren nur noch 13,2 Prozent der Erwerbstätigen im einstigen Agrarland Bayern im primären Sektor tätig. Dagegen stieg die Zahl der Beschäftigten in Industrie und Handwerk bis 1970 auf 47,4 Prozent und im Dienstleistungsbereich auf 39,4 Prozent ${ }^{27}$. Der in den "roaring fifties ${ }^{428}$ einsetzende Strukturwandel prägte die Bundesrepublik nachhaltig. So wurden die einschneidenden Unterschiede zwischen Zentrum und Peripherie, zwischen katholischen und protestantischen Regionen oder zwischen industriell, kleingewerblich und agrarisch geprägten Lebenswelten mehr und mehr eingeebnet, ohne allerdings vollständig zu verschwinden. Für Bayern hat man überspitzt sogar vom „Ende der ,Provinz““ gesprochen $^{29}$.

Die öffentliche Hand hat im Freistaat schon frühzeitig versucht, steuernd in den sozialen und ökonomischen Strukturwandel einzugreifen, etwa durch die Entscheidung, anstatt auf Kohle auf Erdöl und später auf Kernkraft als Energieträger zu setzen, oder durch eine umfassende Landesplanung. Über die Trägerschichten der bayerischen Strukturpolitik ist jedoch nur wenig bekannt. So hat man von informellen, aus Vertretern der CSU, der Ministerialbürokratie und wichtiger Verbände zusammengesetzten "Schaltstellen des Interessenausgleichs" gesprochen, und auch von einem "Gesellschaftspakt zwischen großindustriellen und mittelständischen Kräften“ unter Führung der CSU war die Rede ${ }^{30}$. Es kann allerdings keinen Zweifel daran geben, daß der bayerischen Unionspartei eine zentrale Rolle zufiel; mit Ausnahme der zweiten Amtszeit von Wilhelm Hoegner stellte die CSU seit 1946 stets den Ministerpräsidenten, seit 1957 auch in jedem Kabinett den Wirtschafts- und Finanzminister. Auffällig ist zudem, daß die CSU bereits in den fünfziger Jahren bevorzugt Bundesministerien besetzte, über die sich moderne Technologie nach Bayern transferieren ließ ${ }^{31}$, etwa das Postministerium, das Atomministerium und nicht zuletzt das Verteidigungsministerium. Aber noch kann nichts darüber aussgesagt werden, wie die Bruchlinien in der CSU verliefen, die nie die monolithische Einheit war, als die sie sich selbst gerne präsentier-

\footnotetext{
${ }^{26}$ Hans-Peter Schwarz, Modernisierung oder Restauration? Einige Vorfragen zur künftigen Sozialgeschichtsforschung über die Ära Adenauer, in: Rheinland-Westfalen im Industriezeitalter, Bd. 3: Vom Ende der Weimarer Republik bis zum Land Nordrhein-Westfalen, hrsg. von Kurt Düwell und Wolfgang Köllmann, Wuppertal 1984, S. 278-293, hier S. 289.

27 Vgl. Paul Erker, Keine Sehnsucht nach der Ruhr. Grundzüge der Industrialisierung in Bayern 1900-1970, in: GuG 17 (1991), S. 480-511, hier S. 486 und S. 491.

${ }^{28}$ Schwarz, Modernisierung, in: Rheinland-Westfalen im Industriezeitalter, Bd. 3, S. 291.

${ }^{29}$ Vgl. Heinz Burghart, Das Ende der „Provinz“, in: Georg Jenal (Hrsg.), Gegenwart in Vergangenheit. Beiträge zur Kultur und Geschichte der neueren und neuesten Zeit. Festschrift für Friedrich Prinz zum 65. Geburtstag, München 1993, S. 391-417.

${ }^{30}$ Möckl, Struktur, S. 751, bzw. Mintzel, Geschichte der CSU, S. 259 f.

31 Vgl. Hans-Peter Schwarz, Adenauer. Der Staatsmann: 1952-1967, Stuttgart 1991, S. 353.
} 
te, welche Kräfte die moderne Struktur-, Industrie- und Technologiepolitik trugen und welche sie ablehnten.

Wie die Wahlergebnisse zeigen, hatte der ökonomische und soziale Wandel für die konservative CSU keine negativen Folgen, im Gegenteil. Doch was sich hinter der Formel von der Entwicklung der CSU zur „bayerischen Staats- und Hegemonialpartei ${ }^{\text {“ } 32}$ im einzelnen verbirgt, liegt ebenso im dunkeln wie die Antwort auf die Frage, weshalb die SPD die sich herausbildende Industriearbeiterschaft nicht dauerhaft an sich binden konnte. Lag das vielleicht daran, daß der dramatische Rückgang der Erwerbstätigen in Land- und Forstwirtschaft und die Umschichtung der Betriebsgrößen ein Anwachsen der $\mathrm{Zu}$ - und Nebenerwerbslandwirte mit sich brachte, so daß „ein in den ländlichen Grund und Boden verwurzelter Typ des bayerischen Industriearbeiters" entstehen konnte ${ }^{33}$ ? Gelang der CSU deshalb der schwierige Spagat zwischen ihrer angestammten Anhängerschaft in Landwirtschaft und Handwerk und den Arbeitnehmern im expandierenden sekundären und tertiären Sektor der bayerischen Wirtschaft? Fragen über Fragen also. Für eine theoretisch und methodisch reflektierte, quellengestützte Parteienforschung bleibt gerade im Hinblick auf die bewegten Jahre des Strukturwandels der bayerischen Gesellschaft in den fünfziger und sechziger Jahren noch viel zu tun ${ }^{34}$.

\footnotetext{
${ }^{32}$ Mintzel, Regionale politische Traditionen, in: Oberndörfer/Schmitt (Hrsg.), Parteien und regionale Traditionen, S. 126.

${ }^{33}$ Erker, Keine Sehnsucht nach der Ruhr, S. $499 \mathrm{f}$.

${ }^{34}$ Vgl. dazu Thomas Schlemmer, Gesellschaft und Politik in Bayern 1949-1973. Ein neues Projekt des Instituts für Zeitgeschichte, in: Maximilian Lanzinner, Michael Henker (Hrsg.), Landesgeschichte und Zeitgeschichte. Forschungsperspektiven zur Geschichte Bayerns nach 1945, Augsburg 1997, S. 103-109.
} 